\title{
Carbunco. Prevención de la enfermedad
}

\author{
Anthrax. Prevention of the disease
}

\author{
Bernagozzi, JA¹, Barragán $\mathrm{JH}^{1}$, Anselmino F${ }^{1}$, Bernagozzi ME² \\ ${ }^{1}$ Cátedra de Inmunología II. Facultad de Ciencias Veterinarias, Universidad Nacional de La Plata \\ ${ }^{2}$ Profesional indepediente \\ Correo electrónico del autor: jorgeberna22@gmail.com
}

\begin{abstract}
Resumen:Este trabajo revisa las alternativas para la prevención del carbunco, desde la vacuna desarrollada por Pasteur hasta las actuales. Así, se describen diferentes tipos de vacunas (basadas en el antígeno protector, en esporos inactivados, en plásmidos, entre otras) y los efectos que estas producen.
\end{abstract}

Palabras clave: Bacillus anthracis, factores de patogenicidad, toxinas, inmunopatogenia, vacunas

Abstract: This work reviews the alternatives used for prevention of carbunco, from the vaccine developed by Pasteur up to current ones. Thus, different types of vaccines (based on protective antigen, inactivated spores, or plasmids) and their effects are described.

Key words: Bacillus anthracis, pathogenic factors, toxins, immunopathogenicity, vaccines 


\section{Introducción}

El carbunco, carbunclo bacteridiano, carbón, grano malo o ántrax es una enfermedad conocida desde la antigüedad. Fue mencionada por Hipócrates, quien describió ciertas lesiones cutáneas y le dió el nombre de anthrax, debido a su aspecto (del griego: carbón). Casimir Davaines, médico francés, en 1850, describió por primera vez el agente etiológico al observarlo en la sangre de carneros infectados (Geison, 1995; Kauffman, 2005; Schaible, 2005; Tournier et al., 2009). En 1876, Robert Koch lo aisló en estado puro y lo clasificó, denominándolo Bacillus anthracis (Carter, 1985; Davis et al., 1996; Geison, 1995). Koch, además, entre 1880 y 1881 registró, por primera vez, el uso exitoso de una vacuna a base celular, la que permitió luchar con éxito contra las epizootias producidas por el Bacillus anthracis.

\section{Prevención de la enfermedad: vacunas}

Pasteur, Koch y otros microbiólogos contemporáneos habían observado que cultivos envejecidos o muertos por calor de diversas bacterias, cuando eran inoculados a animales susceptibles, lejos de producir la enfermedad, protegían a éstos de la posterior descarga de las formas patógenas de las mismas (Tizard, 2009). Pasteur aplicó con B. anthracis la misma técnica que utilizaba en la elaboración de otras vacunas bacterianas, sometiendo el bacilo a temperaturas más elevadas de cultivo y obteniendo una cepa modificada que utilizó como vacuna. Erroneamente supuso que la patogenicidad de la misma se había reducido o atenuado. Las vacunas comerciales para prevenir el carbunco datan de 1881 y se basaron en las pruebas realizadas por Pasteur en Pouilly le-Fort con esta cepa "atenuada" por calentamiento (Davis et al., 1996; Geison, 1995).

La primera dosis aplicada a los animales consistía en cultivos de $B$. anthracis atenuados mediante incubación a $42-43^{\circ} \mathrm{C}$, durante $15-20$ días. Esta cepa es conocida como Pasteur I, patógena para ratones y cobayos. La segunda dosis, 2 semanas después, contenía cultivos incubados a $42-43^{\circ} \mathrm{C}$ durante solamente 10-12 días, por lo tanto, menos atenuados. Esta cepa es la denominada Pasteur II, con un grado mayor de patogenicidad que la anterior. Pasteur creyó en ese entonces haber atenuado la patogenicidad de la bacteria. Pero en realidad este mantenimiento de la cepa a temperaturas más elevadas, denominadas "disgenésicas", ocasiona la pérdida de, al menos, diez plásmidos responsables de la producción de antígeno protector (AP), factor edematoso (FE) y factor letal (FL) (Blaustein et al., 1989; Sterne, 1937).

A la luz de los conocimientos actuales podemos afirmar que no se trataba de una verdadera atenuación, sino que, bajo esas condiciones de crecimiento, algunos microorganismos perdían el plásmido con capacidad de producir la toxina, quedando como remanente una baja cantidad de bacilos que lo conservaban y mantenían su capacidad toxigénica intacta. Estos bacilos con aptitud para producir toxinas habrían sido los potenciales inductores de la inmunidad.

Después de las investigaciones de Pouilly leFort, la vacuna de Pasteur adquirió una excelente reputación que determinó que su uso se extendiera ampliamente por Europa y Sudamérica por alrededor de 50 años. Si bien la vacuna propuesta por Pasteur fue una revolución para su época y una solución al problema del carbunco, con el devenir de los años y la masificación de su uso, fueron apareciendo diversos inconvenientes que podemos resumir de esta manera:

a) Dificultad en obtener el nivel de atenuación apropiado para la sensibilidad de cada una de las especies animales a las que se destina.

b) Deterioro y/o modificación del poder patógeno de las cepas por cultivos sucesivos, debido a la alteración lógica de la relación bacterias productoras de toxina vs. no productoras.

c) Esporulación muy pobre en los medios de cultivo líquidos conocidos en la época de Pasteur.

Sin embargo, todas las vacunas elaboradas con esporos atenuados de ese entonces deben considerarse como ligeras modificaciones del método original propuesto por Pasteur.

Una de las primeras modificaciones fue propuesta por Zenkowski en el año 1887, quien recomendó el uso de glicerina para suspender las vacunas esporuladas, con un triple objetivo: inhibir el desarrollo de contaminantes, aumentar la longevidad de los esporos y actuar como adyuvante de la inmunidad. La estandarización y la prueba de eficacia de estas vacunas eran complejas. La prueba de potencia debía realizarse en ovejas, ya que las cepas Pasteur mataban a los cobayos. A los efectos de atenuar o disminuir los efectos deletéreos que producía la vacuna, se recurrió a la aplicación simultánea de vacuna y suero hiperinmune. Esta alternativa, si bien fue utilizada durante bastante tiempo (también utilizada en la vacunación para prevenir la peste porcina clásica), presenta una serie de inconvenientes, que van desde lo económico, ante la necesidad de utilizar suero hiperinmune, hasta la gran dificultad para encontrar el correcto equilibrio y generar una respuesta inmune efectiva sin reacciones adversas. Un exceso de suero disminuye la respuesta inmune pero una escasa cantidad puede provocar reacciones indeseables. Este método fue propuesto por Sorberhein en 1913, quien adicionó suero a la segunda aplicación, es decir, a la cepa menos atenuada.

Durante las décadas de 1920 y 1930, la vacuna 
original sufrió importantes modificaciones. Durante la primera de las décadas mencionadas, a la vacuna original de Pasteur se le adicionó un 50-60 \% de glicerina.

En esta década también se realizaron pruebas con la aplicación de una dosis única de vacuna suspendida en glicerina de una cepa lo suficientemente atenuada que no era patógena para conejos, pero mantenía la patogenicidad para cobayos. Todas las vacunas elaboradas con la cepa Pasteur resultaron muy difíciles de estandarizar.

Entre los años 1929 y 1937 se realizaron distintas investigaciones con diferentes tipos de vacunas, adicionándose entre un 4 y un $10 \%$ de saponina para atenuar la virulencia de la cepa. La saponina es un detergente que provoca una inflamación muy intensa, limitando la diseminación de los esporos, haciendo la vacuna más segura y favoreciendo la inducción de la inmunidad. Sin embargo, es capaz de producir fuertes reacciones inflamatorias y de hipersensibilidad, cuando la pureza de la saponina no es la adecuada.

El método original fue propuesto por Mazuchi en 1931, quien adicionó a la vacuna original entre 2 y $5 \%$ de saponina.

Una de las vacunas más conocidas fue la Carbazoo®, producida por el Instituto Seroterápico Milanés y luego en Estados Unidos por el laboratorio Lederle.

Presumiblemente, se trataba de una vacuna elaborada con una cepa de alta patogenicidad, a la cual se le agregaba saponina en algunos casos hasta en una concentración del $10 \%$, de acuerdo con el criterio de diversos investigadores de la época, entre ellos Sterne, que consideraban que de esta manera se disminuía la patogenicidad de la cepa. Sin embargo, esta concentración de saponina resultaba sumamente tóxica. Esta vacuna tenía la particularidad que debía ser inocua e inofensiva para conejos y cobayos. Posteriormente, Sterne disminuyó la concentración de saponina entre el 0,1 y el $0,5 \%$ para reducir las reacciones indeseables.

Max Sterne, nacido en Italia, en 1905, logró desarrollar una vacuna a partir de una cepa de carbunco patógena en el año 1937 (Sterne, 1937).

La cepa patógena aislada fue cultivada durante 24 horas en un medio que contenía $50 \%$ de suero equino y en una atmósfera con un $30 \%$ de anhídrido carbónico. Obtuvo una cepa rugosa acapsulada y acapsulógena, es decir sin cápsula y sin capacidad de producirla como consecuencia de haber perdido el plásmido responsable de la formación de la misma. Esta nueva cepa fue denominada 34 F2 y la vacuna elaborada a partir de la misma tuvo una amplia difusión en el campo veterinario (Elliot et al., 2000; Shlyakho \& Rubinstein, 1994).
La cepa Sterne ha perdido el plásmido pXO2 responsable de la formación de la cápsula, pero mantiene el pXO1, responsable de la formación de toxina, siendo esta falta de capacidad de la producción de cápsula la única diferencia con la cepa patógena o de campo. Esto determina que la cepa Sterne pueda ser fácilmente endocitada por los neutrófilos (debemos recordar que la cápsula es un componente con propiedades antifagocitarias), aunque mantiene intacta la capacidad de producir toxina en forma similar a la cepa patógena.

En 1944, Sterne comunicó el empleo de su vacuna en gran escala en Sudáfrica. A partir de entonces, el uso de la vacuna avirulenta de Sterne se ha extendido por todo el mundo y en muchos países, como en Argentina, es la única vacuna aprobada por el ente nacional de regulación, SENASA, para su uso en medicina veterinaria.

El éxito obtenido por esta vacuna determinó que se pensara en utilizarla en medicina humana. Dada la capacidad de producción de toxinas que posee la cepa, la vacuna fue calificada como muy agresiva y peligrosa para ser empleada en la especie humana. Sin embargo, y a pesar de ello, fue autorizada y utilizada en numerosas ocasiones en la Unión Soviética, mientras que la mayoría de los países la consideraron inaceptable (Ivins, 1998; Milne et al., 1995; Petosa et al., 1997; Turnbull, 1991).

En 1940, en la Unión Soviética se introdujo una vacuna similar a la Sterne, denominada ST1. Esta cepa, al igual que la Sterne, se considera peligrosa para la especie humana por su capacidad de producir toxinas y por su alta reactogenicidad, además de producir una protección parcial contra el ántrax cutáneo.

La necesidad de desarrollar vacunas efectivas, seguras y no reactogénicas para la especie humana se transformó en una imperiosa necesidad durante los tiempos de la guerra fría ante el peligro cierto del uso del $B$. anthracis como arma biológica (Scorpio et al., 2006).

Durante la década de 1950, se desarrolló una vacuna sin purificar, acelular, a partir de sobrenadante de cultivo, conteniendo el AP, con el objetivo de formar anticuerpos contra este factor y así impedir la penetración en la célula de los otros factores que componen la toxina, el FE y/o el FL. Esta vacuna, de relativa eficacia, aparentemente fue desarrollada casi simultáneamente en el Reino Unido y en los Estados Unidos.

Esta primera generación de vacunas licenciadas en Estados Unidos fue utilizada para inmunizar granjeros, investigadores y personas con alto riesgo de contraer la enfermedad. A pesar de su uso, la eficacia y seguridad de esas vacunas fue cuestionada permanentemente. 
Esta necesidad de conseguir vacunas eficientes y seguras para la prevención de la enfermedad, sobre todo con el objetivo de neutralizar el ántrax contraído por vía inhalatoria, determinó el licenciamiento de vacunas más purificadas, adsorbidas y elaboradas a partir de cepas genéticamente modificadas con incapacidad de producir FE y FL. Las vacunas desarrolladas mediante técnicas de ingeniería genética serán fundamentales para lograr estos objetivos.

Los primeros trabajos sobre la utilización de $B$. anthracis como arma biológica datan de la Primera Guerra Mundial, cuando fue utilizado para matar caballos. Durante la Segunda Guerra Mundial, el ejército japonés desarrolló la primera arma biológica a base de esporos. En la etapa de la posguerra, más precisamente durante la denominada guerra fría, muchos países como Estados Unidos, Gran Bretaña, Canadá e Irak no escatimaron esfuerzos en desarrollar y perfeccionar este tipo de armamento debido a ciertas particularidades del esporo, como son su fácil producción, diseminación y supervivencia que le dan cierta supremacía sobres otros agentes etiológicos como el virus de la viruela humana (small pox) y el virus del Ebola.

La investigación sobre producción de nuevos inmunógenos, que había entrado prácticamente en un período de estancamiento luego del desarrollo de vacunas más seguras, resurgió con vertiginosidad inusitada después de los ataques a las Torres Gemelas el 11 de setiembre de 2001 y de la amenaza de uso de los esporos de ántrax diseminados a través de cañerías de aire acondicionado en edificios y aviones y la contaminación de encomiendas o cartas. Los esporos de Bacillus anthracis inhalados producen una elevada mortalidad, ya que cuando aparecen los signos clínicos, que se confunden primariamente con una gripe, prácticamente es demasiado tarde para instaurar un tratamiento eficaz.

\section{Vacunas basadas en el antigeno protector}

La vacuna para uso humano denominada AVA (Anthrax Vaccine Adsorbed) está basada en el AP adsorbido en hidróxido de aluminio y es utilizada en los Estados Unidos desde hace muchísimos años, siendo más conocida, a partir del año 2002, con el nombre comercial de BioThrax.

Las primeras vacunas de uso humano datan de la década de 1950 en el Reino Unido y desde la década de 1960 en Estados Unidos. Fueron probadas y demostraron su efectividad en la prevención de la infección en muchos modelos animales, incluídos primates no humanos (Hotchkiss et al., 2005).

La vacuna consiste en material sobrenadante de B. anthracis cepa V770-NP1-R. Esta cepa tiene como característica mantener el plásmido pXO1+ productor de toxina y haber perdido el plásmido pXO2- responsable de la presencia de la cápsula, cultivada en forma microaerófila en medios definidos, es decir, medios con una constitución química perfectamente conocida. El sobrenadante del cultivo es filtrado y adsorbido con hidróxido de aluminio. El plan corriente de vacunación consiste en seis dosis inyectadas por vía subcutánea en un término de 18 meses y una revacunación anual (Benson et al., 1998; Brossier et al., 2000; Duesbery et al., 1998; Ivins et al., 1990; Ivins, 1998; Milne et al,. 1995; Petosa et al., 1997; Turnbull et al., 1990; Turnbull, 1991).

Los Estados Unidos disponen, desde 1970, de la denominada AVA, producida por la Bioport Corporation, compuesta, fundamentalmente y como ya hemos consignado, por el AP adsorbido en hidróxido de aluminio, conteniendo, como máximo, 0,83 mg por dosis, adicionado 0,0025 a $0,004 \%$ de cloruro de benzetonio y 0,01 a $0,0037 \%$ de formaldehído, como conservantes. Algunos lotes contienen como impureza algo de FL y menor cantidad de FE. Estimula la inmunidad humoral pero no la celular y se deben administrar 6 dosis a las semanas 0,2 y 4 , y a los 6 , 12 y 18 meses (Brachman et al., 1962; Fellows et al., 2001; Friedlander et al., 1999; Ivins et al., 1992; Ivins et al., 1994; Ivins et al., 1998; Mahlandt et al., 1966; Pittman et al., 2001).

En el Reino Unido se utiliza una vacuna similar, pero proveniente de sobrenadante de la cepa Sterne 34F2. Esta vacuna contiene trazas de FE y FL y es para muchos autores, entre ellos Jones y colaboradores (1996), superior a la norteamericana.

Si bien estas vacunas carecen prácticamente de efectos adversos, y están optimizados, estandarizados y protocolizados los pasos de manufacturación para eliminar los otros componentes de la toxina, existe cierta variabilidad en los procesos, quedando latente la posibilidad de producir reacciones adversas. Esto provocó el desarrollo de una nueva generación de vacunas compuesta por $\mathrm{APr}$ (AP recombinante) purificado de una cepa atoxigénica y aesporogénica de $B$. anthracis. Esta vacuna fue probada experimentalmente en monos Macacus Rhesus, observándose que fue capaz de prevenir la infección por $B$. anthracis inoculados por vía aerógena por medio de aerosoles (Jones et al., 1996; Turnbull, 1991).

Las vacunas a base de cepas con esporas recombinantes parecen ser más eficaces que las obtenidas por formas vegetativas clásicas (Ivins et al., 1986). Cohen y colaboradores en el año 2000 produjeron vacunas con APr. Esta vacuna fue probada en cobayos a los que se les aplicaba una sola dosis de $5 \times 10^{7}$ esporas de la cepa MASC10 con capacidad de expresar, al menos, $100 \mu \mathrm{g}$ por $\mathrm{ml}$ de APr, demostran- 
do protección frente al desafío con cepas patógenas. Esta vacuna, previo análisis de seguridad y eficacia, y luego de obtener autorización de uso por el Instituto de Medicina de la Academia Nacional de Ciencias, EEUU, fue aplicada a las tropas estadounidenses que se desplazaron a la Península Arábiga (Cohen et al., 2000). Algunos informes internacionales atribuyeron a esta vacuna el denominado "síndrome del Golfo". Este síndrome se caracteriza por síntomas inespecíficos y variables como cansancio, dolores musculares y de las articulaciones, jaquecas, pérdida de la memoria y estrés postraumático. Su causa es desconocida y no es mortal, pero puede estar asociada con angustia e incapacidad excesiva, quizás de origen psiquiátrico (Schumm et al., 2002).

Además del desarrollo de estas vacunas de nueva generación, más seguras e inmunogénicas, se están estudiando vías alternativas de inoculación con el objetivo de aumentar la rapidez y eficacia de la respuesta inmune. La vía de inoculación de AVA es la subcutánea. Sin embargo, se están desarrollando experiencias utilizando la vía intramuscular que parece generar menos reacciones indeseables y mayor protección. Esto quizás esté determinado por el daño que el aluminio ocasiona en la fibra muscular, originando la producción de DAMPs (patrones moleculares asociados a daño) capaces de estimular el inflamasoma, sensor citosólico con capacidad para reconocer patrones comunes de los microorganismos, detectar su presencia, es decir la infección, y luego liberar mediadores que favorecen el desarrollo de la respuesta inmune. Tanto la vacuna AVA como la APr han demostrado ser muy eficaces en la producción de $\lg M$ e IgG anti AP. Sin embargo, a efectos de lograr una mejor y más completa protección, es importante desarrollar vacunas que se apliquen por la vía de las mucosas con el objetivo de generar inmunoglobulina A secretoria (IgAs).

\section{Vacunas aplicadas por la vía de las mucosas}

Teniendo en cuenta las tres vías probables de infección con Bacillus anthracis, la vía inhalatoria es, sin duda, la que mayor mortalidad produce, ya que prácticamente carece de sintomatología post infección $y$, por lo general, cuando esta aparece, es demasiado tarde e incluso suele confundirse con cuadros gripales que retrasan la intervención profesional y el tratamiento específico.

La inmunidad humoral circulante cumple un importante rol en la prevención de la enfermedad, pero no de la infección. Prevenir la infección es crucial en la afección respiratoria.

En este sentido, es de vital importancia el desarrollo de IgAs en las mucosas del organismo.
Con este objetivo, se están desarrollando vacunas para generar inmunidad en las mucosas mediante vacunación oral utilizando como vectores Salmonella (S. tiphymurium, S. entérica serovar Typhi) o Lactobacillus genéticamente modificados con capacidad de expresar AP (Coulson et al., 1994; Zegers et al., 1999).

También se encuentran en vía de desarrollo vacunas inoculadas mediante instilación nasal, a base de APr asociado a microesferas que se colocan en la nariz o adsorbidas a liposomas para aumentar la inmunogenicidad. Una variante interesante es la utilización de una vacuna oral elaborada a partir de esporos atenuados de cepas de $B$. anthracis que expresan APr. Se ha desarrollado una vacuna a partir de la cepa Sterne pXO1-, pXO2- transformada, con capacidad de expresar el plásmido de APr (pero no toxinas ni cápsula) e inducir la producción de lgG e lgAs.

Otra vacuna interesante en vía de desarrollo es la que se elabora a partir de lisados de Lactobacillus casei con posibilidad de expresar AP, aplicada por vía enteral y con alta capacidad de respuesta específica.

Trabajos experimentales han demostrado la eficacia de vacunas en cuyo diseño se utilizan adyuvantes como toxina colérica y fosfatidilcolina de soja y otras a base de AP micro encapsulado en esferas de $100 \mathrm{kDa}$ de poli L- lactato. También ha resultado exitosa la utilización de una vacuna a base de esporos de la cepa $\Delta$ Sterne pXO1-, pXO2-, con posibilidad de expresar AP e inducir una respuesta anti AP mediada por IgG e IgAs cuando se la aplica por vía oral a cobayos, siendo capaz de proteger animales vacunados cuando se desafían con $20 \mathrm{DL}_{50}$ de $B$. anthracis. Las ventaja de estas vacunas orales reside en la facilidad de aplicación de las mismas, teniendo en cuenta el plan básico de inmunización que consta, como mínimo, de 6 aplicaciones (Aloni-Gristein et al., 2005; Flick-Smith et al., 2002; Mikszta et al., 2005; Sloat et al., 2005).

\section{Vacunas de doble protección}

Se han elaborado vacunas complementando al APr con antígenos capsulares y con antígenos propios de la superficie del bacilo, como el Bc1A. La idea conceptual es la de tratar de inhibir el desarrollo de formas vegetativas, favoreciendo la fagocitosis por los anticuerpos anti-antígenos capsulares, y evitar la posterior formación de toxinas.

Por otra parte, el AP, además de ser secretado por la forma vegetativa de la bacteria, se encuentra en pequeñas cantidades en la superficie de los esporos sin germinar $y$, por lo tanto, los anticuerpos anti AP pueden unirse a los mismos. Esto disminuye la germinación y favorece la captación por parte de los macrófagos para su destrucción, de acuerdo con lo 
observado in vitro cuando se los enfrenta a cultivos de macrófagos. Si bien es una alternativa válida, otros autores postulan que la presencia de pequeñas cantidades de anticuerpos circulantes puede facilitar, por efecto opsónico, la endocitosis rápida de esporos y así favorecer el desarrollo de la enfermedad, si la tasa de anticuerpos circulantes presentes no permite la neutralización de la toxina a generar.

Considerando la mayor protección ante la descarga de formas patógenas, se obtuvieron resultados satisfactorios inmunizando ratones con vacunas a base de AP, adicionadas con antígenos capsulares y esporos inactivados con formol. Esta vacuna a esporos inactivados es conocida como FIS (formol inactivate spore) (Hahn et al., 2006; Rhie et al., 2003).

\section{Vacunas de esporos inactivados}

En el año 2000, Cohen y colaboradores demostraron que los anticuerpos generados contra los esporos contribuyen a la inmunidad. El trabajo se realizó en cobayos a los que se les inocularon esporos inactivados en suspensión. El AP ligado al esporo en pequeña concentración genera los Ac específicos y facilita la endocitosis y destrucción de los mismos. Es necesario destacar que, para generar respuesta inmune, esta vacuna debe contener una alta carga de esporos dado la escasa concentración del AP en la superficie de los mismos. Otra condición necesaria es que la misma esté adyuvada.

Sin embargo, Brossier y colaboradores, en el año 2002, hicieron lo mismo en conejos, encontrando protección parcial y observando que mejoraba la eficacia de la vacuna cuando se adicionaba AP a los FIS (Brossier et al., 2002; Cohen et al., 2000; Glomski et al., 2005; Steichen et al., 2003).

\section{Vacunas sobre la base de cápsulas}

La obtención de una vacuna con capacidad de neutralizar la propiedad antifagocítica de la cápsula es una alternativa sumamente interesante, ya que permitiría, ni bien el esporo germina, la opsonización, endocitosis y destrucción antes de que la forma vegetativa dé lugar a la producción de las toxinas.

Uno de los problemas a resolver es que la cápsula posee poca capacidad inmunogénica, haciéndose necesario copular o asociar la misma a una proteína que le proporcione capacidad de impacto sobre el sistema inmunológico. Su poca capacidad inmunogénica se debe a su estructura química y a la carga negativa que posee, lo que determina que se comporte, prácticamente, como un antígeno T-independiente (Chabot et al., 2004; Maurer et al., 1965; Schneerson et al., 2003; Wang et al., 2004).

\section{Vacunas de plásmidos}

La inoculación del plásmido codificador del AP al ratón induce la producción de lgG con títulos no muy elevados, pero éstos aumentan rápidamente con la aplicación de un booster o refuerzo a base de APr. Existe una vacuna de diseño elaborada por el USAMRIID (Instituto de Investigaciones Médicas sobre Enfermedades Infecciosas del Ejército de Estados Unidos) que protege al cobayo de la inhalación de esporas (Little et al., 1986).

\section{Otros tipos de vacunas ensayadas}

Pasteur, Chamberland y Roux, en 1883, ensayaron, al menos, tres tipos de vacunas con bacilos muertos con escaso éxito.

En 1956, Schlingman y colaboradores obtuvieron una vacuna a partir de filtrado estéril del sobrenadante rico en AP, purificado y concentrado, con escasa presencia de PE y FL. Varios grupos de investigadores (Puzzis \& Wright, 1954; Darlows et al., 1956; Ivins et al., 1995; Ivins et al., 1998; Firedlander et al.,1999), encontraron que estas vacunas protegían al mono rhesus de la inhalación de esporas. Cuando esta vacuna se inoculó al mono Macacus Rhesus por vía intramuscular se comprobó la formación de lgG e $\lg \mathrm{M}$, y cuando fue aplicada por vía nasal la presencia de IgAs.

Beznosov y colaboradores, en 1997, extrajeron una proteína de $92 \mathrm{kD}$ de la forma vegetativa de la cepa de $B$. anthracis STI-1 atenuada, utilizando lauril-sarcosilato sódico al $3 \%$ y a $4^{\circ} \mathrm{C}$, y purificada mediante cromatografía de absorción en hidroxiapatita y adicionada con adyuvante de Freund. Fue probada con éxito en ratones (Beznosov et al., 1997).

Todas estas vacunas necesitan estar adyuvadas para aumentar su capacidad de impacto, utilizándose, entre otros, hidróxido de aluminio, monofosforil lípido A, Bordetella pertussis y Corynebacterium ovis, lo que aumenta las reacciones vacunales.

Se ha ensayado para animales el adyuvante denominado Detox, compuesto por las paredes de Mycobacterium phlei o de Mycobacterium bovis, ricas en muramildipéptido (MDP) más $10 \%$ de monofosforil lípido $A(M P L)$ y $2 \%$ de escualeno.

El triple mix o Tri Mix es un adyuvante que utiliza como base el Detox más dimicolato de trealosa y emulsión de MPL unido a escualeno-lecitina-tween 80. Brussière y colaboradores, en 1995, utilizaron este tipo de adyuvante y demostraron que la aplicación de esta vacuna puede causar uveítis y artritis.

Estos adyuvantes no están autorizados para ser usados en vacunas para seres humanos, y si bien no figuran en los protocolos de composición de las vacunas empleadas en los soldados, en varios cientos 
de afectados por el síndrome de la Guerra del Golfo se encontraron anticuerpos antiescualeno, haciendo pensar en su uso secreto (Nass, 1998).

Cuando se desarrollan vacunas, el desafío primario es conseguir una buena inmunogenicidad y una baja reactogenicidad. Además, deben ser seguras e incapaces de revertir la patogenicidad (si se trata de cepas atenuadas) y de no provocar reacciones adversas graves.

El desafío actual para los investigadores es desarrollar vacunas que, además de las propiedades citadas, sean capaces de brindar protección ante cepas cuya patogenicidad sea modificada intencionalmente para ser utilizadas en la guerra biológica, con el agravante, además, de que estas cepas pueden poseer resistencia a los antibióticos.

El desarrollo de nuevas vacunas de diseño fue posible a partir del conocimiento de la estructura proteómica de $B$. anthracis. Identificar las proteínas que el bacilo expresa específicamente o preferentemente durante la infección, o aquellas que contribuyan a la virulencia, será seguramente de vital importancia en el desarrollo de nuevas vacunas.

En este aspecto, debemos resaltar los esfuerzos en desarrollar vacunas con nuevos antígenos, utilizando como base subunidades multivalentes. El inconveniente que presentan este tipo de vacunas, como todas las de subunidades, es que precisamente, por tratarse de fracciones antigénicas del bacilo, se pierde capacidad de impacto sobre el sistema inmune y requieren adyuvantes muy importantes para el reconocimiento escalonado a través de los PRRs.

Sin embargo, cuando se compara la eficacia de la protección conferida por una vacuna a base de esporas vivas como la cepa Sterne con la conferida por vacunas de subunidades, nos encontramos que, en general, es mayor la protección brindada por la esporovacuna, a pesar de que la de subunidades genera una buena respuesta anti AP. En 1986, Little y Knudson encontraron que la vacuna Sterne confirió una protección del $80 \%$ frente a la descarga de 1000 esporos, mientras que la de subunidades brindó solamente una protección del $50 \%$ en las mismas condiciones. Casi con seguridad podemos afirmar que esta diferencia en la eficacia a favor de la cepa Sterne se debe a la formación de cantidades relativas de FE y FL que, aunque indeseables desde el punto de vista de la reactogenicidad, colaboran grandemente en los fenómenos de protección a campo (Ivins et al., 1998).

Cuando se compara la respuesta de distintas vacunas se debe tener muy en cuenta el sistema de evaluación utilizado. Cuando se realizan evaluaciones solamente in vitro, como por ejemplo cuantificación de anticuerpos, se dejan de lado otros factores que hacen a la patogenicidad in vivo y los resultados pueden ser disímiles y quizás una vacuna que in vitro denota una excelente producción de anticuerpos específicos no nos brinde, in vivo, una protección adecuada.

En la actualidad, a través de la ingeniería genética, se ha logrado insertar dentro de especies del genero Bacillus, particularmente $B$. subtilis, que no posee la capacidad natural de producir toxinas, el gen capaz de codificar y hacer expresar AP (Ivins et al., 1986). Se han elaborado vacunas a partir de este AP y se logró producir protección en el cobayo.

La protección inducida por vacunas elaboradas a partir de AP producido por $B$. amyloliquefaciens fue mucho más eficiente que la obtenida a partir de $B$. subtilis.

Se ha obtenido AP atóxico utilizable como vacuna a partir de Lactobacillus casei y también a partir de $B$. subtilis lisogenizado por el fago phi 105.

EI USAMRIID obtuvo una vacuna a base de una mutante de la cepa Sterne, cuya supervivencia depende de ciertos cuerpos aromáticos. Estos cuerpos aromáticos necesarios para la supervivencia del bacilo no se encuentran presentes en los tejidos humanos. Por lo tanto, cuando es utilizada como vacuna, esta cepa tiene la particularidad de reproducirse, aunque en forma limitada, y no tiene capacidad de difundir e invadir el organismo.

Las vacunas desarrolladas a partir de cepas con mutaciones que afectan la distribución de las mismas en el hospedador pueden resultar muy peligrosas, ya que se ha determinado que son muy inestables y pueden revertir a la forma patógena. No obstante, las nuevas técnicas de secuenciación y mutagénesis pueden hacer más eficiente y segura la producción de estas vacunas.

El desarrollo de nuevas vacunas exige el cumplimiento de estrictos protocolos que garanticen la eficacia y la falta de reactogenicidad.

\section{Condiciones requeridas para los ensayos de vacunas}

Cuando se realizan estudios en los que se compara la eficiencia de dos o más vacunas, los mismos deben realizarse en sistemas biológicos fiables que produzcan resultados similares (Belton et al., 1956; Brachman et al., 1945; Pittman et al., 2002).

En general, las vacunas de uso en medicina humana son probadas, en cuanto a su seguridad, con distintos modelos en primates no humanos. Generalmente, el ratón y el cobayo son especies difíciles de proteger cuando se descargan cepas totalmente virulentas de $B$. anthracis. El tipo de ratón utilizado es crucial en las pruebas, ya que la susceptibilidad varía grandemente según las líneas genéticas. Un estudio comparó diez líneas celulares encontrando variantes 
sustanciales en la $D_{50}$ y en el tiempo necesario para producir la enfermedad, variando éste desde 3 a 6,5 días.

También se encuentran importantes diferencias con relación a la $\mathrm{DL}_{50}$ cuando se desafían con cepas atenuadas. Por ejemplo, algunas cepas de ratones como la $A / J$ son sensibles a una $\mathrm{DL}_{50}$ de $1,1 \times 10^{3}$ esporos de la cepa Sterne, mientras otras cepas, como la $\mathrm{C} 58 / \mathrm{J}$, son resistentes a una descarga de esta misma cepa con más de $10^{7}$ esporos. Las mismas diferencias se encontraron con respecto a la $\mathrm{DL}_{50}$ de esporos y cuando se utilizó la descarga de toxinas. Por ejemplo, la cepa de rata denominada Fisher 344 es muy sensible a FL y es, por ende, la más comúnmente usada. De la misma manera que ciertas cepas de ratas muestran una sensibilidad extrema a las toxinas, paradójicamente resultan muy difíciles de infectar, mientras que otros animales, como los cobayos, son muy resistentes a las toxinas y pueden morir cuando se las infecta con una escasa cantidad de esporos. La razón de esta discrepancia no está muy bien estudiada, pero parece ser que la diferencia radica en la capacidad del esporo para germinar en el hospedador, en la capacidad de expresión de los receptores de AP, en la respuesta inmune y en la sensibilidad a las toxinas.

Uno de los métodos más comúnmente utilizados in vitro para evaluación de vacunas es el método de ELISA, fundamentalmente para medir anticuerpos antitóxicos o anticápsula.

También se utilizan métodos de seroneutralización, sobre todo anti-FL, mediante los cuales los macrófagos murinos son protegidos in vitro con antisuero anti FL purificado. Este método de seroneutralización es tomado como gold estándar para evaluar respuestas a vacunas anti AP, aunque algunos estudios posteriores demostraron que estos títulos no guardan correlación exacta con la protección in vivo.

La medición de la inmunidad mediada por células no se utiliza, probablemente porque $B$. anthracis es un patógeno de vida extracelular y cuya patogenicidad está ligada fundamentalmente a la acción tóxica.

Probablemente, la evaluación de las nuevas vacunas requerirá de la combinación de pruebas más complejas que incluyan respuesta de anticuerpos, neutralización de toxinas, muerte de bacterias e inmunidad celular.

\section{Vacuna Sterne: generación de respuesta inmune}

La vacuna de uso veterinario contra carbunco bacteridiano cepa Sterne es la única vacuna aprobada por el SENASA para utilización en la República Argentina.

Es una vacuna a base de esporos, clasificada técnicamente como esporovacuna, que contiene, como mínimo, 10.000.000 de esporas por mililitro, suspendidas en partes iguales de solución salina bufferada y glicerina, adicionada de saponina como coadyuvante de la inmunidad.

Como es sabido, la característica esencial de la cepa Sterne, es de ser acapsulada y acapsulógena, pero mantiene la capacidad de producir los tres factores componentes de su única toxina: el AP, FE y el FL.

Prácticamente, la única diferencia entre la cepa Sterne y el $B$. anthracis patógeno, comúnmente denominado cepa de campo, es la falta de cápsula, ya que la capacidad de producir toxinas es desde el punto de vista cuali-cuantitativo, prácticamente la misma.

La enfermedad a campo se produce cuando los esporos de $B$. anthracis ingresan al organismo, encuentran las condiciones ideales de germinación y pasan a la forma vegetativa capsulada. La cápsula tiene como función principal impedir la fagocitosis y así escapar a los mecanismos de destrucción por los PMN para poder continuar su desarrollo y elaborar, finalmente, la toxina responsable de la muerte del animal.

Cuando se inocula la vacuna Sterne la saponina presente en la formulación produce un fenómeno inflamatorio severo en el sitio de inoculación. Como en todo proceso inflamatorio la tensión parcial de oxígeno es menor que en los tejidos normales. Esta microaerofilia favorece el pasaje de la forma esporulada de resistencia a la forma vegetativa carente de cápsula y así es susceptible de ser fagocitada y eliminada por los PMN. De esta manera, el organismo va eliminando la misma pero una pequeña cantidad de bacilos que escapa a los mecanismos fagocíticos va liberando limitadas cantidades de toxinas con capacidad de estimular el sistema inmune, lo que determinará la generación de una respuesta antitóxica que protegerá de la enfermedad.

Los anticuerpos antitóxicos son los principales responsables de la protección, teniendo en cuenta que estamos ante una septicemia, siendo las toxinas liberadas la principal causa de muerte. Hasta el momento, no se ha puesto énfasis sobre la inmunidad mediada por células, pero es indudable que la misma debe tener injerencia en algún proceso intermedio $y$, como mencionan recientes trabajos, en procesos colaborativos en la prevención y/o desarrollo de la enfermedad (Ingram et al., 2013).

Si bien esta secuencia de acontecimientos es comprensible y lógica, creemos que es demasiado superficial y que no contempla algunas particularidades que tiene esta bacteria cuando es analizada a la luz de los conocimientos actuales.

Cuando estudiamos el fenómeno con más pro- 
fundidad, surgen algunas dudas y nos permitimos presuponer que la realidad es algo diferente a la expuesta.

El primer interrogante que cabe preguntarse es la siguiente:

Si la cepa vacunal tiene la capacidad de producir la misma cantidad de toxina que la cepa patógena y la generada por ésta provoca supresión de la respuesta inmune innata, afectando PMN, CD, LB y macrófagos, es dable suponer que la misma toxina producida por la cepa vacunal debería provocar un efecto similar sobre las células del sistema inmune.

Es más, cuando se realizan pruebas de seguridad sobre la vacuna terminada y estandarizada en cobayos se observa que el peso que, según la técnica, debe ser de 550 a $600 \mathrm{~g}$, es determinante para la supervivencia de los animales. Si utilizamos cobayos de menor peso, por ejemplo, de $250 \mathrm{~g}$, es probable que ninguno de ellos sobreviva.

Por otra parte, cuando la vacuna, por error en la estandarización, supera las 15.000 .000 de esporas por $\mathrm{ml}$, se hace necesario realizar pruebas complementarias en ovejas para demostrar la seguridad. También existen referencias de que algunos terneros de 3 a 4 meses que por error fueron vacunados con el doble de la dosis $(84 \mathrm{ml})$, han muerto como consecuencia de la vacunación.

Esto es indicativo de que es una vacuna con una significativa capacidad tóxica potencial.

Todo esto nos llevó a profundizar la investigación sobre los reales mecanismos inmunitarios que despliega la vacuna. No hemos encontrado en la bibliografía información precisa que permita dilucidar la cuestión.

Según nuestro punto de vista, el mecanismo inmune estaría mediado por una serie de sucesos:

1.- Se ha demostrado que no todas las CD o macrófagos son sensibles a la toxina. Algunas de estas células se ven afectadas por ella, mientras que otras del mismo individuo son resistentes. Es más, dentro de la misma especie, existen razas que son resistentes a la enfermedad, precisamente por no verse afectadas estas células.

2.- Diversos autores, a través de comunicaciones personales, nos han referido que aparentemente el primer factor que el bacilo secreta es el AP y, posteriormente, los otros dos. Esta aseveración nos permite postular que este AP puede ser captado por las CD en una etapa temprana, para ser procesado, presentado y transportado al linfonódulo para iniciar la respuesta inmune. Debemos recordar que las CD a partir de su origen en la médula ósea, tienen alta capacidad fagocítica con muy baja o nula expresión de moléculas de coestímulo, como CD80/86 y CMH, moléculas que se adquieren a través de su proceso madurativo. A partir del momento en que toman contacto con un antígeno, tal como el AP, migran hacia el linfonódulo y dan origen a los procesos de inducción de la respuesta inmune, para que las células plasmáticas elaboren los primeros anticuerpos antitóxicos.

3.- Tampoco debemos olvidar que en la generación de la respuesta inmune interviene una gran cantidad de $C D$, macrófagos y linfocitos $B$ y que algunos de ellos pueden ser resistentes a la acción tóxica e inducir respuesta, mientras que otros sucumben a la toxina.

4.- El AP presente en el esporo, a pesar de hallarse en una pequeña cantidad, puede, al ser ingerido por el macrófago, iniciar una respuesta inmune temprana específica.

De acuerdo con nuestro criterio, sería relevante la hipótesis de que el factor AP es liberado prontamente $\mathrm{y}$, en primer término, lo que permitiría el procesamiento, la presentación y el transporte al linfonódulo de los epitopes específicos, iniciando la respuesta inmune contra éste.

Estas CD ya no se verían afectadas por la producción de los otros factores, FE y FL.

Cuando el proceso infeccioso avanza y estos factores son liberados con posterioridad al AP, las poblaciones de CD y macrófagos responsables de la inducción de la respuesta inmune sensibles a la toxina se verán afectadas y no podrán ejercer su función específica.

Las poblaciones celulares no sensibles a la toxina no se verían afectadas por las mismas y desarrollarían sus funciones propias, originando la inmunidad antitóxica. A medida que se van liberando pequeñas cantidades de Ac específicos, estos se unen al AP libre formando inmunocomplejos, los que son opsónicamente endocitados. Esto favorece la formación y liberación de nuevos Ac antitóxicos.

El trabajo recientemente publicado por Ingram et al. (2013) demuestra la importancia que poseen los receptores específicos de unión de la toxina a la célula, no sólo en la enfermedad, sino en la respuesta inmune a vacunas. Estos autores demostraron que la exposición previa a pequeñas cantidades de toxina modula negativamente la expresión de los receptores (ANTXR) y, por consiguiente, la captación del AP, lo que provoca la iniciación del proceso tóxico cuando se unen los FE o FL.

Trabajos publicados con anterioridad analizando tres tipos de poblaciones humanas: naturalmente infectados, vacunados y no expuestos, demostraron una notable variación de expresión de los receptores específicos en sus leucocitos (Maldonado-Arocho et al., 2006; Warfel et al., 2005). Analizando los porcentajes de ANTXR1 presente en los monocitos de donantes voluntarios de sangre, encontraron grandes 
diferencias en la expresión de los receptores específicos, por lo que clasificaron a estas poblaciones en grupos denominados de "baja" o "alta" capacidad de expresión. Estos resultados establecen notables diferencias de sensibilidad hacia la toxina en líneas de células linfoblásticas humanas, como resultado, en parte, de los niveles de expresión de ANTXR2 (Martchenko et al., 2012).

Trabajando con padres e hijos, descubrieron, además, que la variedad de expresión está ligada a procesos genéticos. Estos hallazgos confirman, de manera contundente, la presencia de células sensibles y otras refractarias a la toxina, detallando, además, el fundamento de su comportamiento. Estos resultados permitieron a los autores postular la existencia de una estrecha correlación entre la susceptibilidad a los efectos de la toxina en modelos animales in vivo e in vitro y la elevada expresión de receptores ANTXR1 (Martchenko et al., 2012; Salles et al., 2006; Young et al., 2007). Los individuos con alta capacidad de expresión de receptores poseen una elevada susceptibilidad a la infección, como así también al desarrollo de una enfermedad mucho más severa (Doganay et al., 2010; Warfel et al., 2005).

Estos estudios fueron realizados con pacientes que habían padecido ántrax cutáneo y recibido tratamiento antibiótico. Curiosamente, los individuos recuperados presentaban baja capacidad de expresión de los receptores. Esta reducción de la expresión de receptores podría estar relacionada con la elevada persistencia de toxina en los individuos infectados.

Está demostrado que el FL produce activación del inflamasoma de las células afectadas determinando la activación del sistema de las caspasas y liberación de citoquinas proinflamatorias, originando la muerte rápida de los macrófagos por piroptosis (Muehlbauer et al., 2007; Popov et al., 2002; Terra et al., 2010). Por lo tanto, es posible que mediante este mecanismo puedan haberse eliminado muchas de las células que expresan ANTXR1 en la población de individuos que han estado expuestos previamente y por largo tiempo a la toxina, sobreviviendo aquellas células con baja expresión de receptores. Se ha sugerido que esta activación inducida por la LT del NIrp1b (inflamasoma) y la posterior y consecuente muerte de los macrófagos puede ser una respuesta inmune innata de protección, en oposición a mecanismos de virulencia utilizados por $B$. anthracis (Terra et al., 2010).

Las personas vacunadas reciben una exposición primaria intensa al AP, componente principal de la vacuna. Las concentraciones de FE y FL son muy bajas y puede afirmarse que estos dos factores son "contaminantes" indeseados pero presentes en el inmunógeno, a pesar de los procesos de purificación de esta vacuna. Consecuentemente, estos individuos están expuestos a una cantidad muy inferior de toxinas que los infectados.

También quedó demostrado que el marco inflamatorio del proceso, vacunal o infeccioso, juega un rol muy importante en la expresión de receptores. Sin ninguna duda, el correspondiente a la infección natural es diferente al provocado por la vacunación.

Los monocitos de los individuos no expuestos previamente tienen una gran capacidad de fijar AP más allá de si se encuentran clasificados en los grupos con "baja" o "alta" capacidad de expresión de receptores. Esto, sin ninguna duda, está asociado a la co-expresión de ANTXR2 y/o beta1 integrinas en los monocitos. Si bien no fue posible en el estudio evaluar cantidades de AP ligados a las células, quedó claramente demostrado que una reducción de los niveles de presencia de ANTXR1 provoca una reducción considerable de macrófagos que han adherido AP.

El receptor ANTXR2 ha demostrado tener una mayor afinidad para ligar AP que el ANTXR1 (Scobie et al., 2003). Sin embargo, existen sustanciales diferencias en relación con el pH del sitio donde se produce la adherencia. Así, los receptores ANTXR2 prefieren un $\mathrm{pH}$ ácido de 5 a 6 , mientras que los ANTXR1 lo hacen a pH más fisiológico, entre 6 y 8 (Fu et al., 2010).

Analizada en conjunto, queda claro que la reducción del porcentaje de monocitos que expresan ANTXR1 podría tener un papel fundamental en la respuesta del huésped a las toxinas de ántrax.

También ha quedado demostrada la importancia, casi desconocida hasta el momento, de la producción de IFN-y por parte de las células T específicas anti AP provocando una disminución de la expresión en los monocitos de los receptores ANTXR1. Por otra parte, modelos murinos han sugerido que el IFN-y originado como respuesta de las células T CD4 es protector frente a las esporas de ántrax (Muehlbauer et al., 2007).

Los resultados y conclusiones presentadas parecen tener implicancias relevantes en lo que hace a la eficacia de la vacuna. Sin embargo, se hacen necesarios posteriores estudios acerca de la correlación de los niveles de unión de AP, la expresión de los ANTXRs, las respuestas de células T y los títulos de anticuerpos.

Dado el papel desempeñado por ANTXRs en la susceptibilidad a la toxina del ántrax, (Salles et al., 2006; Young et al., 2005) la relación inversa entre el porcentaje de monocitos que expresan ANTXR1 y la magnitud de las respuestas de células $T$, cualquier desarrollo de vacuna o adyuvante que sea capaz de regular negativamente la expresión de los ANTXR podría resultar beneficioso para la efectividad de la misma. 
Se requieren futuras investigaciones que permitan desentrañar el mecanismo íntimo de inducción de la inmunidad, la función específica de linfocitos $\mathrm{T}$, la producción de INF-y y la protección de los individuos.

\section{Carbunco en Argentina}

Las primeras citas referentes a la aparición de carbunco en la República Argentina podemos encontrarlas en las actas del Cabildo de Buenos Aires entre los años 1590 y 1609.

El Dr. Francisco Javier Muñiz (1795-1871) médico y científico argentino, describió un caso humano de la enfermedad en el año 1847.

En 1859, Luis Pasteur, conocedor de la problemática que representaba el carbunco en la República Argentina, envió numerosas dosis de la vacuna por él elaborada para la prevención de la enfermedad.

Con posterioridad, José Hernández en su publicación del año 1882 mencionó el carbunco como "el grano, enfermedad conocida desde tiempos muy antiguos" e indíca la necesidad de no desollar animales muertos de manera inesperada.

En 1886 se registró fehacientemente la vacunación con vacuna Pasteur en una estancia de Gualeguay, Entre Ríos.

El Dr. Bidali fue uno de los pioneros e impulsores de la elaboración de la vacuna en el país, pues habiendo trabajado con Pasteur comprendió la necesidad imperiosa de prevenir la enfermedad que para ese entonces provocaba grandes mortandades de bovinos, como asimismo la transmisión del carbunco a las personas involucradas en tareas rurales.

En la República Argentina fueron utilizadas en forma rutinaria durante muchísimos años las vacunas Pasteur, denominadas Pasteur I y Pasteur II. También fue utilizada una cepa denominada Chaco, probablemente de patogenicidad intermedia entre las Pasteur I y II, de difícil caracterización, la denominada Delphy 5 y la cepa Sterne.

Mediante la Resolución 705/81, SENASA estableció las normas de elaboración, control y uso de la cepa Sterne. La Ley N ${ }^{\circ}$ 6703/1961 y la Resolución 115/2014 establecieron la obligatoriedad de la vacunación en la provincia de Buenos Aires. La provincia de Santa Fe, mediante la Resolución № 1007/14 vigente, estableció el "Plan de Vacunación obligatorio" contra carbunco bacteridiano.

Por otra parte, las autoridades de Salud Pública Nacional acordaron recomendar a la Comisión Provincial de Sanidad Animal (COPROSA) la implementación de un plan superador de lucha contra el carbunco que incluya la vacunación obligatoria en conjunto con la fiebre aftosa. También se establece la figura de "Veterinario Corresponsable Sanitario".
El Ministerio de Agroindustria de la Provincia de Buenos Aires, mediante la Resolución 115/2014, estableció una serie de medidas que pueden consultarse en la página de la institución: http://www.maa. gba.gov.ar/2010/SubPED/Ganaderia/carbunclo.php.

Desde el año 2002 a la fecha, el Dr. Ramón Noseda y el Laboratorio Azul emiten informes y alertas de casos de carbunco rural en la Provincia de Buenos Aires, habiéndose creado el "Área de evaluación de carbunco rural en la provincia de Buenos Aires" constituida por 30 partidos de la misma.

EI Dr. Noseda, en su informe anual correspondiente al año 2015, presentó los resultados de la evaluación en tres áreas de vacunación anticarbuncosa simultánea con aftosa:

a) Área de Alerta y Respuesta (partido de Azul, provincia de Buenos Aires). En 2014 se vacunó el $85 \%$ de la población blanco, mientras que en 2015 se vacunó el $68 \%$, es decir, 17 puntos porcentuales menos de bovinos vacunados.

b) Provincia de Buenos Aires. Según datos no oficiales, en la campaña de 2014 se vacunó el $83 \%$ de la población blanco estimada, mientras que, en 2015 , solamente se vacunó el $63 \%$, es decir, 20 puntos porcentuales menos.

c) Provincia de Santa Fe. Durante 2014 se vacunó el $73 \%$ de su población blanco expuesta mientras que, en 2015 , se logró vacunar el $86 \%$, es decir, 13 puntos porcentuales más que el año anterior.

\section{Conclusiones}

$B$. anthracis es un patógeno con características muy particulares, responsable de provocar una grave enfermedad septicémica en diversas especies animales y en el hombre. Por otra parte, constituye un arma biológica utilizada desde la Primera Guerra Mundial, cobrando inusitada importancia en los ataques bioterroristas con posterioridad a la caída de las Torres Gemelas en el año 2001.

Desde los trabajos de Pasteur en el siglo XIX se han desarrollado vacunas elaboradas a partir de distintas variantes de la cepa de campo patógena hasta las noveles vacunas desarrolladas mediante ingeniería genética. Todas apuntan a un denominador común: vacunas muy eficaces, seguras y carentes de reacciones adversas. Sin embargo, nuevos desafíos asoman en el horizonte. La posibilidad de que se utilicen en bioterrorismo cepas genéticamente modificadas y/o resistentes a antibióticos, hace necesario el desarrollo de vacunas que generen una respuesta inmune cuali-cuantitativamente superior, con capacidad de neutralizar esas posibles mutaciones genéticas del bacilo. Además, deberían ser capaces de inducir IgAs a efectos de prevenir la infección, sobre todo 
para proteger contra la presentación respiratoria de la enfermedad, forma elegida preferentemente para los ataques biológicos.

Las vacunas destinadas a la especie humana han sido el objetivo principal del desarrollo tecnológico, conociéndose perfectamente el mecanismo de acción y de protección generado por la vacuna.

En medicina veterinaria, prácticamente, no se ha avanzado mucho desde la aparición de la vacuna a cepa Sterne. Esta vacuna, si bien en líneas generales es considerada como segura e inmunogénica, conlleva un riesgo potencial que es la generación de todos los componentes de la toxina, con acción deletérea sobre el sistema inmune.

Creímos oportuno indagar sobre los mecanismos inmunogénicos intrínsecos de la misma. No hemos encontrado muchas explicaciones que vayan más allá de la tradicional académica. Como resultado de estas pesquisas y de comunicaciones personales con autores de relevancia y reconocidos en la materia, nos permitimos, a modo de presunción, elaborar la teoría desarrollada up supra.

Creemos que resultaría de interés generar, a partir de la presente revisión bibliográfica, un foro de discusión que contribuya al conocimiento sobre el real mecanismo inmunogénico de las distintas vacunas disponibles.

\section{Bibliografía}

Aloni-Gristein R, Gat O, Altboum Z, Velan B, Cohen S, Shafferman A. 2005. Oral spore vaccine based on live attenuated nontoxigenic Bacillus anthracis expressing recombinant mutant protective antigen. Infection and Immunity. 73:4043-53. doi: 10.1128/IAI.73.7.4043-4053.2005.

Belton FC, Darlow HM, Henderson DW. 1956. The use of anthrax antigen to immunise man and monkey. Lancet. 271(6941):476-9.

Benson EL, Huynh PD, Finkelstein A, Collier RJ. 1998. Identification of residues protective antigen channel. Biochemistry. 37:3941-8. doi: 10.1021/bi972657b.

Beznosov MV, Petrov GA, Sorkin I, Filippova OL, Rogov SN. 1997. The isolation of the surface antigen from vegetative cells of Bacillus anthracis STI-1 and study of its protective properties. Zhurnal Mikrobiologii, Epidemiologii, i Immunobiologii. 1:9-13.

Blaustein RO, Koehjer TM, Collier RJ, Finkelstein A. 1989. Anthrax toxin: channel-forming activity of protective antigen in planar phospholipoid bilayers. Proceedings of the National Academy of Sciences of the United States of America. 86:2209-13.

Brachman PS, Gold H, Plotkin SA, Fekety RF, Werrin M, Ingraham NR. 1945. Field evaluation of human anthrax vaccine. American Journal of Public Health Nations. 52(4):632-45.

Brachman PS, Gold H, Plotkin SA, Fekety FR, Werrin M, Ingraham NR. 1962. Field evaluation of a human anthrax vaccine. American Journal of Public Health. 19:3241-7.
Brossier F, Weber Levy M, Mock M, Sirard JC. 2000. Role of toxin functional domains in anthrax pathogenesis. Infection and Immunity. 68:178-186.

Brossier F, Levy M, Mock M. 2002. Anthrax spores make an essential contribution to vaccine efficacy. Infection and Immunity. 70:661-4.

Bussière JL, Mc Cormick GC, Green JD. 1995. Preclinical safety assesment considerations. Pharmaceutical Biotechnology. 6:61.

Carter KC. 1985. Koch's postulates in relation to the work of Jacob Henle and Edwin Klebs. Medical History. 29:353-74.

Chabot D J, Scorpio A, Tobery SA, Little SF, Norris SL, Friedlander AM. 2004. Anthrax capsule vaccine protects against experimental infection. Vaccine. 23:43-7. doi: 10.1016/j. vaccine.2004.05.029.

Cohen S, Mendelson I, Altboum Z, Kobiler D, Elanany E, Bino T, Leitner M, Inbar I, Rosemberg H, Gozes H, Gozes Y, Barak R, Fisher M, Kronman C, Velan B, Shafferman A. 2000. Attenuated nontoxigenic and noncapsulated recombinant Bacillus anthracis spore vaccines protects against anthrax. Infection and Immunity. 68:4549-58. doi: 10.1128/ IAI.68.8.4549-4558.2000.

Coulson NM, Fulop M, Titball RW. 1994. Bacillus anthracis protective antigen, expressed in Salmonella typhimurium SL 3261 , affords protection against anthrax spore challenge. Vaccine. 12:1395-401.

Davis B, Dulbecco R, Herman N, Eisen H, Ginsberg H, 1996. Tratado de microbiología. 4a Ed. Barcelona. Salvat Editores.

Darlow HM, Belton FC, Henderson DN. 1956. The use anthrax antigen to immunize man and monkey. Lancet. 271:476-9.

Doganay M, Metan G, Alp E. 2010. A review of cutaneous anthrax and its outcome. Journal of Infection and Public Health 3:98-105. doi: 10.1016/j.jiph.2010.07.004.

Duesbery NS, Webb CP, Leppla SH, Gordon VM, Klimpel KR, Copeland TD, Ahn NG, Oskarsson MK, Fukasawa K, Paul KD, Vande Woude GF. 1998. Proteolytic inactivation of MAPkinase-kinase by anthrax lethal factor. Science. 280:734-7.

Elliot JL, Mogridge J, Collier RJ. 2000. A quantitative study of the interaction of Bacillus anthracis edema factor and lethal factor with activated protective antigen. Biochemistry. 39:6706-13. doi: 10.1021/bi000310u.

Fellows PF, Linscott MK, Ivins BE, Pitt ML, Rossi CA, Gibbs $\mathrm{PH}$, Friedlander AM. 2001. Efficacy of a human anthrax vaccine in guinea pigs, rabbits, and rhesus macaques against challenge by Bacillus anthtracis isolates of diverse geographical origin. Vaccine. 19:3241-7.

Flick-Smith HC, Eyles JE, Hebdon R, Waters EL, Beedham RJ, Stagg TJ, Miller J, Alpar HO, Baillie LW, Williamson ED. 2002. Mucosal or parenteral administration of microsphereassociated Bacillus anthracis protective antigen protects against anthrax infection in mice. Infection and Immunity. 70:2022-8. doi: 10.1128/IAI.70.4.2022-2028.2002.

Friedlander AM, Pittman PR, Parker GW. 1999. Anthrax vaccine: evidence for safety and efficacy against inhalational anthrax. Journal of the American Medical Association. 282:2104-6.

Fu S, Tong X, Cai Ch, Zhao Y, Wu Y, Li Y, Xu J, Zhang XC, Xu L, Chen W, Rao Z. 2010. The structure of tumor endothelial 
marker 8 (TEM8) extracellular domain and implications for its receptor function for recognizing anthrax toxin. PloS ONE 5, e11203. doi: 10.1371/journal.pone.0011203.

Geison GL. The private science of Louis Pasteur. 1995. Princeton, Princeton University Press.

Glomski IJ, Kepler SJ, Corre JP, Mock M, Goossens PL. 2005. Spores confer protective cellular immunity against capsulated Bacillus anthracis. ACT. Conference 2005. Santa Fe, EE.UU.

Hahn UK, Boehm R, Beyer W. 2006. DNA vaccination against anthrax in mice-combination of anti-spore and anti-toxin components. Vaccine. 24:4595-7. doi: 10.1016/j. vaccine.2005.08.031.

Hotchkiss KA, Basile CM, Spring SC, Bonuccelli G, Lisanti MP, Terman BI. 2005. TEM 8 expression stimulates endothelial cell adhesion and migration by regulating cellmatrix interactions on collagen. Experimental Cell Research. 305:133-44. doi: 10.1016/j.yexcr.2004.12.025.

Ingram RJ, Harris A, Ascough S, Metan G, Doganay M, Ballie L, Williamson ED, Dyson H, Robinson JH, Sriskandan S, Altmann DM. 2013. Exposure to anthrax toxin alters human leucocyte expression of anthrax toxin receptor 1. Clinical and Experimental Immunology. 173:84-91. doi: 10.1111/ cei.12090.

Ivins BE, Ezzell JW Jr, Jemski J, Hedlund K, Risttroph JD, Leppla SH. 1986. Immunization studies with attenuated strains of Bacillus anthracis. Infection and Immunity. 52:454-8.

Ivins BE, Welkos SL. 1986. Cloning and expression of the Bacillus anthracis protective antigen gene in Bacillus subtilis. Infection and Immunity. 54:537-42.

Ivin BE, Welkos SL, Knudson GB, Little SF. 1990. Immunization against anthrax with aromatic complex dependent (Aro-) mutants of Bacillus anthracis and with recombinant strain of Bacillus subtilis that produce anthrax protective antigen. Infection and Immunity 58:303-8.

Ivins BE, Welkos SL, Little SF, Knudson GB. 1990. Cloned protective activity and progress in development of improved anthrax vaccines. Salisbury Medical Bulletin, 68 (suppl), 86.

Ivins BE, Fellows PF, Nelson GO. 1994. Efficacy of standard human anthrax vaccine against Bacillus anthracis spore challenge in guinea pigs. Vaccine. 12, 872.

Ivins BE, Fellows PF, Pitt L, Estrep J, Farchaus J, Friedlander A, Gibbs P. 1995. Experimental anthrax vaccines: efficacy of adjuvants combined with protective antigen against an aerosol Bacillus anthracis spore challenge in guinea pigs. Vaccine. 13(18):1779-84.

Ivins BE. 1998. Anthrax vaccines. How stable is the potency? The ASMth ifc General Meeting. Atlanta, EE. UU. May 18.

Ivins BE, Pitt ML, Fellows PF, Farchaus JW, Benner GE, Waag DM, Little SF, Anderson, GW Jr, Gibbs PH, Friedlander AM. 1998. Comparative efficacy of experimental anthrax vaccine candidates against inhalation anthrax in rhesus macaques. Vaccine. 16:1141-8.

Jones MN, Beedham RJ, Turnbull PC, Fitzgeorge RB, Manchee RJ. 1996. Antibiotic prophylaxis for inhalations anthrax. Salisbury Medical Bulletin 87, (suppl) 127.

Kauffman SH, Schaible UE. 2005. 100th anniversary of Robert Koch's Nobel Prize for the discovery of the tubercle bacillus. Trends in Microbiology. 13(10), 469-75. doi: 10.1016/j.tim.2005.08.003.

Little SF, Knudson GB. 1986. Comparative efficacy of Bacillus anthracis live spore vaccine and protective antigen vaccine against anthrax in the guinea pig. Infection and Immunity. 52:509-12.

Mahlandt BG, Klein F, Lincoln RE, Haines BW, Jones WI Jr, Friedman RH. 1966. Immunologic studies of anthrax. IV. Evaluation of the immunogenicity of three components of anthrax toxin. Journal of Immunology. 96:727-33.

Maldonado-Arocho FJ, Fulcher JA, Lee B, Bradley KA. 2006. Anthrax oedema toxin induces anthrax toxin receptor expression in monocyte-derived cells. Molecular Microbiology. 61:324-37. doi: 10.1111/j.1365-2958.2006.05232.x.

Martchenko M, Candille SI, Tang H, Cohen SN. 2012. Human genetic variation altering anthrax toxin sensitivity. Proceedings National Academic Sciences USA, 109:2972-7.

Maurer PH. 1965. Antigenicity of polypeptides (poly alpha amino acids) XIII. Immunological studies with synthetic polymers containing only D- or D- and L- $\alpha$-amino acids. Journal of Experimental Medicine. 121:339-49.

Mikszta JA, Sullivan VJ, Dean C, Waterston AM, Alarcon JB, Dekker JP 3rd, Brittingham JM, Huang J, Hwang CR, Ferriter M, Jiang G, Mar K, Saikh K U, Stiles BG, Roy CJ, Ulrich RG, Harvey NG. 2005. Protective immunization against inhalational anthrax: a comparison of minimaly invasive delivery platforms. Journal of Infectious Diseases. 191:278-88.

Milne JC, Blanke S R, Hanna PC, Collier RJ. 1995. Protective antigen- binding domain of anthrax lethal factor mediates translocation of a heterologous protein fused to its amino-orcarboy-terminus. Molecular Microbiology. 15:661-6.

Muehlbauer SM, Evering TH, Bonuccelli G, Squires RC, Ashton AW, Porcelli SA. 2007. Anthrax lethal toxin kills macrophages in a strain-specific manner by apoptosis or caspase-1-mediated necrosis. Cell Cycle. 6:758-66. doi: 10.4161/cc.6.6.3991.

Nass M. 1998. Anthrax vaccine safety and efficacy: response to Army Surgeon General Ronald Blanck's posting.

Petosa C, Collier RJ, Klimpel KR, Leppla SH, Liddington RC. 1997. Crystal structure of the anthrax protective antigen. Nature. 385:833-8.

Pittman PR, Gibbs PH, Cannon TL, Friedlander AM. 2001. Anthrax vaccine: short term safety experience in humans. Vaccine. 20:972-8. doi: 10.1016/S0264-410X(01)00387-5.

Pittman PR, Kim-Ahn G, Pifat DY, Coon K, Gibbs P, Little S, Pace-Templeton J, Myers R, Parker GW, Friedlander AM. 2002. Anthrax vaccine: immunogenicity and safety of a dosereduction, route-change comparison study in humans. Vaccine. 20(9-10):1412-20. doi:10.1016/S0264-410X(01)00462-5.

Popov SG, Villasmil R, Bernardi J, Grene E, Cardwell J, Wu A, Alibek D, Bailey C, Alibek K. 2002. Lethal toxin of Bacillus anthracis causes apoptosis of macrophages. Biochemical and Biophysical Research Communications. 293:349-55.

Puziss M, Wright GG. 1954. Studies on immunity in anthrax. IV. Factors influencing elaboration of the protective antigen of Bacillus anthracis in chemically defined media. Journal of Bacteriology 68(4):474-82.

Rhie GE, Roehrl MH, Mourez M, Collier RJ, Mekalanos JJ, Wang JY. 2003. A dually active anthrax vaccine that confers protection against both bacilli and toxins. Proceedings of 
the National Academy of Sciences of the United States of America. 100:10925-30.

Salles II, Voth DE, Ward SC, Averette KM, Tweten RK, Bradley KA, Ballard JD. 2006. Cytotoxic activity of Bacillus anthracis protective antigen observed in a macrophage cell line overexpressing ANTXR1. Cellular Microbiology. 8:1272-81.

Scobie, H. M. and Young, J. A. T. 2005. Interactions between anthrax toxin receptors and protective antigen. Current Opinion in Microbiology. 8:106-12.

Schneerson R, Kubler-Kielb J, Liu TY, Day ZD, Leppla SH, Yergey A, Backlund P, Shiloach J, Majadrly F, Robbins JB. 2003. Poly (gamma-D-glutamic acid) protein conjugates induce lgG antibodies in mice to the capsule of Bacillus anthracis; a potencial addition to the anthrax vaccine. Proceedings of the National Academy of Sciences of the United States of America. 100:8945-50.

Schumm WR, Webb FJ, Jurich AP, Bollman SR. 2002. Comments on the Institute of Medicine's 2002 report on the safety of anthrax vaccine. Psychological Reports. 91:187-91.

Scobie HM, Young JA. 2005. Interactions between anthrax toxin receptors and protective antigen. Current Opinion in Microbiology. 8:106-12. doi: 10.1016/j.mib.2004.12.005.

Scobie HM, Rainey GJA, Bradley KA, Young JA. 2003. Human capillary morphogenesis protein 2 functions as an anthrax toxin receptor. Proceedings of the National Academy of Sciences of the United States of America. 100:5170-4.

Scorpio A, Blank TE, Day WA, Chabot D J. 2006. Anthrax vaccines: Pasteur to the present. Cellular and Molecular Life Sciences. 63:2237-48. doi: 10.1007/s00018-006-6312-3

Shlyakhov EN, Rubinstein E. 1994. Human live anthrax vaccine in the former USSR. Vaccine. 12(8):727-30.

Sloat BR, Cui Z. 2005. Strong mucosal and sistemic immunities induced by nasal immunization with anthrax protective antigen protein incorporated in liposome-protamine-DNA particles. Pharmaceutical Research. 23:262-9.

Steichen C, Chen P, Kearney JF, Turnbough CL Jr. 2003. Identification of the immunodominant protein and other proteins of the Bacillus anthracis exosporium. Journal of Bacteriology. 185:1903-10.

Sterne M. 1937. Avirulent antrax vaccine. Onderstepoort Journal of Veterinary Science and Animal Industry. 7:41-3.

Terra JK, Cote CK, France B, Jenkins AL, Bozue JA, Welkos SL. 2010. Cutting edge: resistance to Bacillus anthracis infection mediated by a lethal toxin sensitive allele of Nalp $1 \mathrm{~b} /$ Nirp1b. Journal of Immunology 184:17-20. doi: 10.4049/ jimmunol.0903114.

Tizard IR. 2009. Inmunología Veterinaria. 8va Ed. Elsevier. España.

Tournier JN, Ulrich RG, Quesnel-Hellman A, Mohamadzadeh M, Stiles BG. 2009. Anthrax, toxin and vaccines: a 125-year journey targeting Bacillus anthracis. Expert Review of Anti-Infective Therapy. 7 (2):219-36. doi: 10.1586/14787210.7.2.219.

Turnbull PC, Quin CP, Hewson P, Stockbridge M, Melling J. 1990. Protection conferred by microbially supplement UK and purified PA vaccine Salisbury Medical Bulletin. 68 (suppl), 89.

Turnbull PC. 1991. Anthrax vaccine: past, present and future. Vaccine. 9(8):533-9.
Wang TT, Fellows PF, Leighton TJ, Lucas AH. 2004. Induction of opsonic antibodies to the gamma-D-glutamic acid capsule of Bacillus anthracis by immunization with a synthetic peptide-carrier protein conjugate. FEMS Immunology and Medical Microbiology. 40:231-7. doi: 10.1016/ S0928-8244(03)00366-3.

[Warfel JM, Steele AD, D’Agnillo F. 2005. Anthrax lethal toxin induces endothelial barrier dysfunction. American Journal of Patholology. 166:1871-81. doi: 10.1016/S00029440(10)62496-0.

Young JJ, Bromberg-White JL, Zylstra C, Church JT, Boguslawski E, Resau JH, Williams BO, Duesbery NS. 2007. LRP5 and LRP6 are not required for protective antigenmediated internalization or lethality of anthrax lethal toxin. PLOS Pathogens 3:27. doi: 10.1371/journal.ppat.0030027.

Zegers ND, Kluter E, van Der Stap H, van Dura E, van Dalen P, Shaw M, Baillie L. 1999. Expression of the protective antigen of Bacillus anthracis by Lactobacillus casei: towards the development of an oral vaccine against anthrax. Journal of Applied Microbiology. 87:309-14. 